No. 12(19)

\title{
REMARKS ABOUT THE SQUARE EQUATION. FUNCTIONAL SQUARE ROOT OF A LOGARITHM
}

\section{Arkadiusz Maciuk, Antoni Smoluk}

\begin{abstract}
The study shows that the functional equation $f(f(x))=\ln (1+x)$ has a unique result in a semigroup of power series with the intercept equal to 0 and the function composition as an operation. This function is continuous, as per the work of Paulsen [2016]. This solution introduces into statistics the law of the one-and-a-half logarithm. Sometimes the natural growth processes do not yield to the law of the logarithm, and a double logarithm weakens the growth too much. The law of the one-and-a-half logarithm proposed in this study might be the solution.
\end{abstract}

Keywords: functional square root, fractional iteration, power series, logarithm, semigroup.

JEL Classification: C13, C65.

DOI: $10.15611 /$ me.2016.12.04.

\section{Introduction}

If the natural logarithm of random variable has normal distribution, then one considers it a logarithmic law - with lognormal distribution. If the function $f$ being function composition to itself is a natural logarithm, that is $f(f(x))=\ln (1+x)$, then the function $g(x)=f(\ln (x))$ applied to a random variable will give a new distribution law, which - similarly as the law of logarithm - is called the law of the one-and-a-half logarithm.

A square root can be defined in any semigroup $G$. A semigroup is a nonempty set with one combined operation and a neutral element. The general form of a quadratic equation has the form of

$$
x^{2}=a \text {, }
$$

where $a \in G$. Solving such an equation is equivalent to answering the question when the element $a$ is a square in semigroup $G$. When the semigroup $G$ is a set of functions with the function composition as an operation and identity function as a neutral element, the solution $f$ of the quadratic equation $f^{2}(x)=f \circ f(x)=g(x)$ is called a functional square root of function $g$.

\footnotetext{
Arkadiusz Maciuk, Antoni Smoluk

Department of Mathematics and Cybernetics, Wrocław University of Economics

arkadiusz.maciuk@ue.wroc.pl
} 


\section{Examples}

If $G$ is a semigroup of natural numbers $\mathbb{N}=\{0,1, \ldots\}$ with addition, then every even number $n=2 k$ is a square, as the quadratic equation is satisfied when $x=k, k+k=n$. A square in this case represents a simple parity of natural numbers. If, however, $\mathbb{N}$ is a semigroup of natural numbers with the operation of multiplication, then the quadratic equation boils down to the question: which natural numbers are real squares. In this case, the answer is a little more difficult. The equation $x^{2}=n$ has a solution if and only if in every prime number in the decomposition of the number $n$ into prime factors occurs an even number of times. Zero is a square because $0=0 \times 0$. One is a square because $1=1 \times 1$, two is not a square, neither is three, but four is because $4=2 \times 2$. The number 36 is because $36=2^{2} \times 3^{2}$, and so on. If $G$ is a semigroup of rational numbers $\mathbb{Q}$ with the operation of addition, each rational number $a \in \mathbb{Q}$ is a square, because $a=\frac{1}{2} a+\frac{1}{2} a$, thus the number $x=\frac{1}{2} a$ is the solution. If $G$ is a semigroup of $\mathbb{Q}$ with the operation of multiplication, then the answer to the question of which rational numbers are squares is more complex. The irreducible fraction $\frac{m}{n}$, where $m, n \in \mathbb{N}$ is a square if and only if both the numerator and denominator are squares of natural numbers. The fraction $\frac{4}{25}$ is a square, however $\frac{12}{25}$ is not. Of course, numbers smaller than zero are not squares, but 0 is a square. If the semigroup $G$ is a set of real numbers $\mathbb{R}$ with addition, then similarly as in semigroup $\mathbb{Q}$ each number is a square. If this semigroup is considered with the operation of multiplication, then any non-negative number $a$ is a square because $a=\sqrt{a} \sqrt{a}$, and negative numbers are not squares. In semigroup $Z_{3}=\{0,1,2\}$ of residues modulo 3 with addition, each element is a square because $0=0+0,1=2+2,2=1+1$. The same set with the operation of multiplication has other squares: $0=0 \times 0,1=1 \times 1=2 \times 2$, but 2 is not a square.

If the semigroup $G$ is a set of real polynomials Pol (F) with the operation of superposition, then in the semigroup squares are polynomials of degree zero, each monomial of the form $f(x)=a x+b$, where $a \geq 0$ is a square. The prerequisite for a polynomial to be a square, is for its degree to be a square in a semigroup of natural numbers with multiplication. 


\section{Generalizations}

Theoretically, the semigroup of words $W(X)$ in the alphabet $X$ is important. The product of the word $S=x y$ and $T=a b$ is a word $S T=x y a b$. The empty word denoted by 0 is a neutral element: $0 S=S 0=S$. The word $S$ is a square if and only if $S=T T$. The prerequisite of being a square is the appearance of each letter in the word an even number of times. The word $T=a b b a$ satisfies a necessary condition but it is not a square, and the word $S=b a b b a b$ satisfies a necessary condition and is a square, $S=T T$, where $T=b a b$. The equation $T^{2}=S$ is easily solved; there is always one solution or the equation is contradictory.

A quadratic equation can be generalized. Let $m, n \in \mathbb{N}$ and $a \in G$. One looks for element $x$ that in semigroup $G$ satisfies the condition $x^{m}=a^{n}$. A special case of this equation is the equation $x^{2}=a$. The solution of this equation in some cases, as in the above examples, is almost trivial. If the semigroup $G$ is a set of functions defined in the domain $X$ transforming $X$ into itself and the operation in this group is function composition, then the solution to a quadratic equation becomes a difficult problem. If the set $G$ is a set of permutations, this equation $x^{2}=\sigma$ does not have solutions when $\sigma$ is an odd permutation, i.e. when its character is number -1 ; setting any permutation with each other always gives an even permutation. The answer to the question: for which permutations this equation has a solution, is not obvious.

What is existence in mathematics? This is a declaration that a certain object exists, i.e. the axiom of existence. Generally, we can say that in the set $\mathbb{A}$ there is an object that satisfies the predicate $\varphi$, if the given below logical sentence is true:

$$
\exists(a \in \mathbb{A}) \wedge \varphi(a) .
$$

An absolute axiom of existence is considered to be true if its adoption does not lead to a conflict of theory. Theorems of existence, as the one above, are conditional in nature: if the axioms are true, then the resulting theorems are also true.

In a semigroup of real functions of one variable with the submission as the operation, the equation $f^{2}(x)=g(x)$ where the function $g(x)$ is given, can have an infinite number of solutions, and may not have a solution at all. For example, there might be an indefinite number of solutions to equation $f^{2}(x)=x$, i.e. involution. Involution is the identity function, function $f(x)=-x$, hyperbole $f(x)=x^{-1}$ for $x \neq 0$, and 0 for $x=0$ but also functions like function $f(x)=x+1$ for $x \in(0,1], f(x)=x-1$, for $x \in(1,2]$ 
and $f(x)=x$ the remaining arguments. In contrast, the solution of equation $f^{2}(x)=x^{-1}$ does not exist [Kuczma 1969]. Bödewart [Bödewart 1944; by Zhang 1997] showed that if function $g: I=[a, b] \rightarrow I$ is continuous and strictly increasing, then for any integer $n>1$ and $A, B \in(a, b)$ with $A<B$ equation $f^{n}(x)=g(x)$ has a continuous and strictly increasing solution $f$ on I satisfying $g(a) \leq f(A)<f(B) \leq g(b)$. It is easy to show that this solution is unambiguous [Iga]. Hence equations such as $f^{2}(x)=e^{x}$ or $f^{2}(x)=\ln (x)$ have a clear unambiguous solution, which is a continuous and strictly increasing function. In 1950 for the first time a method for solving the equation $f^{2}(x)=e^{x}$ was proposed [Kneser 1950], and to this day this equation has lived to see several different methods of solution [Paulsen 2016].

\section{Breakdown method}

Our goal is to solve the equation $f(f(x))=\ln (x)$ which we will further refer to as the Kopociński equation, from the author of the question of whether the natural logarithm in analytic functions' semigroup is a square and what its analytical range is.

The result is the well-known fact that the functional square root of function $e^{x}-1$ is an analytic function in the neighborhood of zero [see Paulsen 2016]. Hence the functional square root of function $\ln (1+x)$, as an inverse function of this function, is also an analytic function in the neighborhood of zero. To find its analytical sequence, we can use one of several methods. Examination of the function $g(x)=\ln (1+x)$ instead of the function $\ln (x)$ is beneficial due to the fact that in its point 0 this function takes the value 0 . The function develops into the power series

$$
g(x)=\sum_{n=0}^{\infty} a_{n} x^{n}
$$

where $a_{n}=\frac{g^{(n)}(0)}{n !}$. Because, $g^{(0)}(0)=0$ and $g^{(n+1)}(0)=(-1)^{n} n$ !, so $g(x)=\sum_{n=0}^{\infty} \frac{(-1)^{n}}{n+1} x^{n+1}$ hence $g(x)=x-\frac{1}{2} x^{2}+\frac{1}{3} x^{3}-\cdots$, for $|x|<1$. One looks for a function $f(x)=b_{0}+b_{1} x+\cdots$ analytic in the neighborhood of zero and satisfying the equation $f(f(x))=x-\frac{1}{2} x^{2}+\frac{1}{3} x^{3}-\cdots$. The composition of real analytic functions is a real analytic function to solve a quadratic equation to calculate the coefficients $b_{n} \in \mathbb{R}$ of the function $g$. 
Symbol $\mathbb{K}$ indicates a semigroup of power series with operation of function composition. This semigroup includes analytic functions at zero, in particular function $g(x)=\ln (1+x)$.

Lemma. If the power series satisfies a quadratic equation in semigroup $\mathbb{K}$, then coefficient $b_{0}=0$.

Evidence. Le $h=f$ o $f$, where $f$ meets the quadratic equation in semigroup $\mathbb{K}$. Of course $h(0)=0$, because $g(0)=0$. It follows that $h(0)=$ $f\left(b_{0}\right)=0$. Zero is thus a fixed point function $h$; also $b_{0}$ a fixed point of function $h$ for $h\left(b_{0}\right)=f\left(f\left(b_{0}\right)\right)=f(0)=b_{0}$. This function $g$ has exactly one fixed point, hence $b_{0}=0$ because $h$ here is the same as $g$, which completes the proof.

We have

$$
\begin{gathered}
f(f(x))=b_{0}+b_{1}\left(b_{0}+b_{1} x+b_{2} x^{2}+\cdots\right)+ \\
b_{2}\left(b_{0}+b_{1} x+b_{2} x^{2}+\cdots\right)^{2}+\cdots=\sum_{n=0}^{\infty} \frac{(-1)^{n}}{n+1} x^{n+1}
\end{gathered}
$$

The lemma shows that

$$
\begin{gathered}
h(x)=b_{1}\left(b_{1} x+b_{2} x^{2}+\cdots\right)+b_{2}\left(b_{1} x+b_{2} x^{2}+\cdots\right)^{2}+ \\
b_{3}\left(b_{1} x+b_{2} x^{2}+\cdots\right)^{3}+\cdots=x-\frac{1}{2} x^{2}+\frac{1}{3} x^{3}+\cdots,
\end{gathered}
$$

so $b_{1}^{2}=1$ because $f^{\prime}(0)=1$. Hence $b_{1}=1$ or $b_{1}=-1$. If $b_{1}=1$ then $h(x)=x+b_{2} x^{2}+\cdots+b_{2}\left(x+b_{2} x^{2}+\cdots\right)^{2}+\cdots=x-\frac{1}{2} x^{2}+\frac{1}{3} x^{3}+\cdots$; we obtain equality $b_{2}+b_{2}=-\frac{1}{2}$, that is $b_{2}=-\frac{1}{4}$. If the above equality $b_{1}$ had the value of -1 then $h(x)=x-b_{2} x^{2}-\cdots+b_{2}\left(-x+b_{2} x^{2}+\cdots\right)^{2}+\cdots=$ $x-\frac{1}{2} x^{2}+\frac{1}{3} x^{3}-\cdots$, that is $0=-b_{2}+b_{2}=-\frac{1}{2}$, which is contradictory. Other factors are calculated recursively.

The expression $\left(b_{1} x+b_{2} x^{2}+\cdots+b_{n} x^{n}+\cdots\right)^{n}$ is a polynomial wherein the value of the $k$-th power of the variable $x$, for $k \geq n$, depends on the number of possible breakdowns of the number $k$ into $n$ numbers. The breakdown of a of a natural number $k \geq 1$ is a finite sequence of natural numbers different from zero summing up to $k$. Number 1 has one breakdown (1), number 2 has two $(1,1)$ and (2). Number 3 has four breakdowns $(1,1,1),(1,2),(2,1)$ and $(3)$, and so on. 
Each of these breakdowns corresponds to $n$-th element permutation with number repetitions whose sum is $k$. For example, for $=4$ and $k=8$ there are

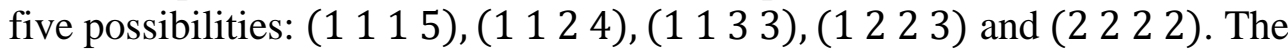
breakdown (1 115$)$ occurs 4 times, breakdown (1 1 33 ) 6 times,

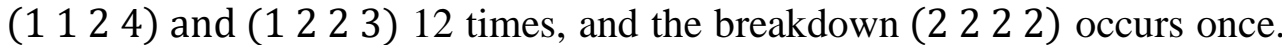
Hence, after expanding $\left(\sum_{i=1}^{\infty} b_{i} x^{i}\right)^{4}$ the coefficient $x^{8}$ of is equal $4 b_{1}^{3} b_{5}+$ $12 b_{1}^{2} b_{2} b_{4}+6 b_{1}^{2} b_{3}^{2}+12 b_{1} b_{2}^{2} b_{3}+b_{2}^{4}$.

In order to determine the formula for the coefficient at the $k$-th power of function $f$ it is sufficient to consider all the possible breakdowns of the number $k$, whereas the coefficient determined by a one-element breakdown is multiplied by $b_{1}$, the coefficient determined by a two-element breakdown is multiplied by $b_{2}$, and so on. For example, the number 2 can be represented as a breakdown composed of a single element 2 or in the form of $(1,1)$ it is the coefficient at $x^{2}$ and has the form of $b_{1} b_{2}+b_{2} b_{1}^{2}$. Breakdown 3 is respectively $3,(1,2),(1,1,1)$ which means that the coefficient at $x^{3}$ is equal to $b_{1}\left(b_{3}\right)+b_{2}\left(2 b_{1} b_{2}\right)+b_{3}\left(b_{1}^{3}\right)=b_{1} b_{3}\left(1+b_{1}^{2}\right)+2 b_{1} b_{2}^{2} ; 4$ is $4,(1,3),(2,2)$, $(1,1,2),(1,1,1,1)$, hence the coefficient at $x^{4}$ is $b_{1}\left(b_{4}\right)+b_{2}\left(2 b_{1} b_{3}+b_{2}^{2}\right)+$ $b_{3}\left(b_{1}^{2} b_{2}\right)+b_{4}\left(b_{1}^{4}\right)=b_{1} b_{4}\left(1+b_{1}^{3}\right)+2 b_{1} b_{2} b_{3}+b_{2}^{3}+b_{1}^{2} b_{2} b_{3}$. And so on, comparing the thus obtained polynomial of coefficients $b_{i}$ to the corresponding values of the expansion of the Maclaurin series of the function $\ln (1+x)$ one obtains:

$$
\begin{gathered}
\left(b_{1}+b_{1}^{2}\right) b_{2}=-\frac{1}{2} \\
2 b_{1} b_{2}{ }^{2}+\left(b_{1}+b_{1}^{3}\right) b_{3}=\frac{1}{3} \\
b_{2}{ }^{3}+2 b_{1} b_{2} b_{3}+3 b_{1}{ }^{2} b_{2} b_{3}+\left(b_{1}+b_{1}^{4}\right) b_{4}=-\frac{1}{4} \\
2 b_{2}{ }^{2} b_{3}+3 b_{1} b_{2}{ }^{2} b_{3}+3 b_{1}{ }^{2} b_{3}{ }^{2}+2 b_{1} b_{2} b_{4}+4 b_{1}{ }^{3} b_{2} b_{4}+\left(b_{1}+b_{1}^{5}\right) b_{5}=\frac{1}{5} .
\end{gathered}
$$

This system is solved recursively:

$$
\begin{gathered}
b_{1}=1, \quad b_{2}=-\frac{1}{4}, \quad b_{3}=\frac{1}{b_{1}+b_{1}^{2}}\left(\frac{1}{3}-\phi_{2}\left(b_{1}, b_{2}\right)\right)=\frac{1}{2}\left(\frac{1}{3}-\frac{1}{8}\right)=\frac{5}{48} \\
b_{4}=\frac{1}{b_{1}+b_{1}^{3}}\left(-\frac{1}{4}+\phi_{3}\left(b_{1}, b_{2}, b_{3}\right)\right)=\frac{1}{2}\left(-\frac{1}{4}+\frac{7}{48}\right)=-\frac{5}{96} \\
b_{5}=\frac{1}{b_{1}+b_{1}^{4}}\left(\frac{1}{5}-\phi_{4}\left(b_{1}, b_{2}, b_{3}, b_{4}\right)\right)=\frac{1}{2}\left(\frac{1}{5}-\frac{55}{384}\right)=\frac{109}{3840} .
\end{gathered}
$$


Generally we have: $b_{1}=1, b_{n+1}=\frac{1}{2}\left(\frac{(-1)^{n}}{n+1}-\phi_{n}\left(b_{2}, b_{3}, \ldots, b_{n}\right)\right)$. These calculations show that:

$$
\begin{aligned}
f(x)=x-\frac{x^{2}}{4} & +\frac{5 x^{3}}{48}-\frac{5 x^{4}}{96}+\frac{109 x^{5}}{3840}-\frac{497 x^{6}}{30720}+\frac{127 x^{7}}{13440}-\frac{11569 x^{8}}{2064384} \\
& +\frac{312757 x^{9}}{92897280}-\frac{1219255 x^{10}}{594542592}+\frac{165677473 x^{11}}{130799370240} \\
& -\frac{885730939 x^{12}}{1121137459200}+\frac{20163875141 x^{13}}{40809403514880} \\
& -\frac{252312616027 x^{14}}{816188070297600}+\frac{9565074633871 x^{15}}{48971284217856000} \\
& -\frac{691138954263097 x^{16}}{5484783832399872000}+\frac{5061676927076641 x^{17}}{62160883433865216000} \\
& -\frac{95993669516238563 x^{18}}{1918107260244983808000} \\
& +\frac{1245671625068799013 x^{19}}{41650329079605362688000} \\
& -\frac{903291663542261320331 x^{20}}{40817322498013255434240000}+\cdots \approx \\
& -0.104167 x^{3}-0.0520833 x^{4}+0.0283854 x^{5} \\
& +0.0033667 x^{9}-0.00205074 x^{10}+0.00126665 x^{11} \\
& -0.000790029 x^{12}+0.000494099 x^{13} \\
& -0.000309135 x^{14}+0.00019532 x^{15}-0.00012601 x^{16} \\
& +0.0000814287 x^{17}-0.000050046 x^{18} \\
& +0.0000299078 x^{19}-0.0000221301 x^{20} .
\end{aligned}
$$

Figure 1 shows a polynomial $f_{20}(x)$ which is the sum of the first twenty terms of a power series $f$ together with functions $\ln (1+x)$ and function $f_{20}^{3}(x)=$ $\ln \left(1+f_{20}(x)\right)$ for the open interval $(-1,1)$. 


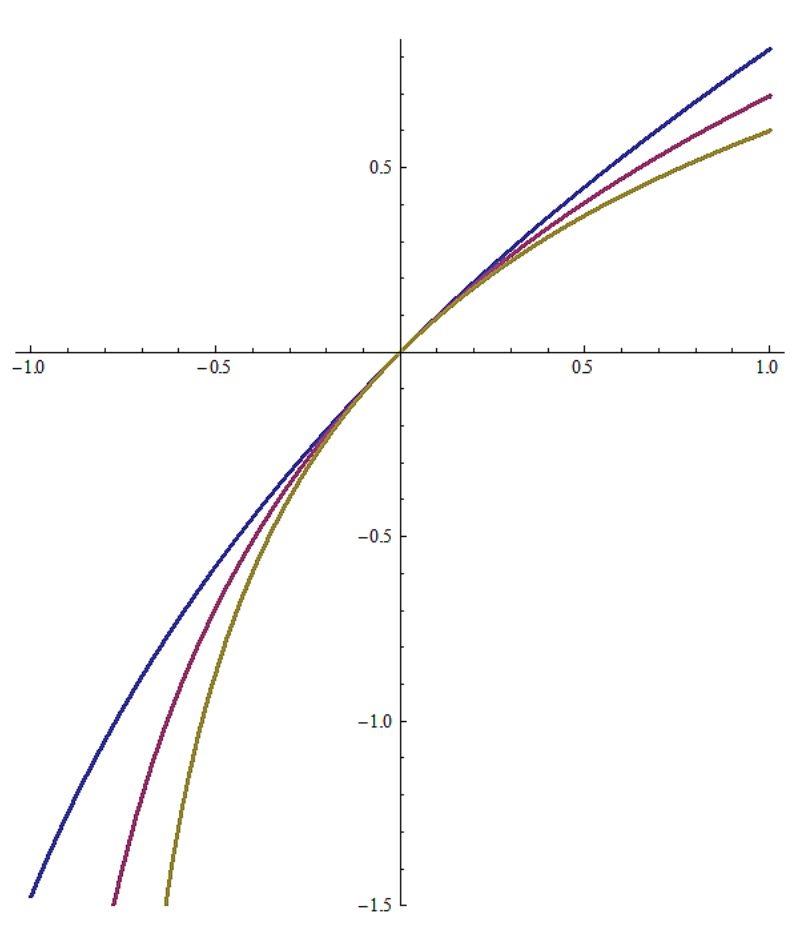

Fig. 1. Selection of function graphs $f(x)$ represented by a polynomial $f_{20}(x)$, function $\ln (1+x)$ and function $\ln \left(1+f_{20}(x)\right)$

Source: own elaboration.

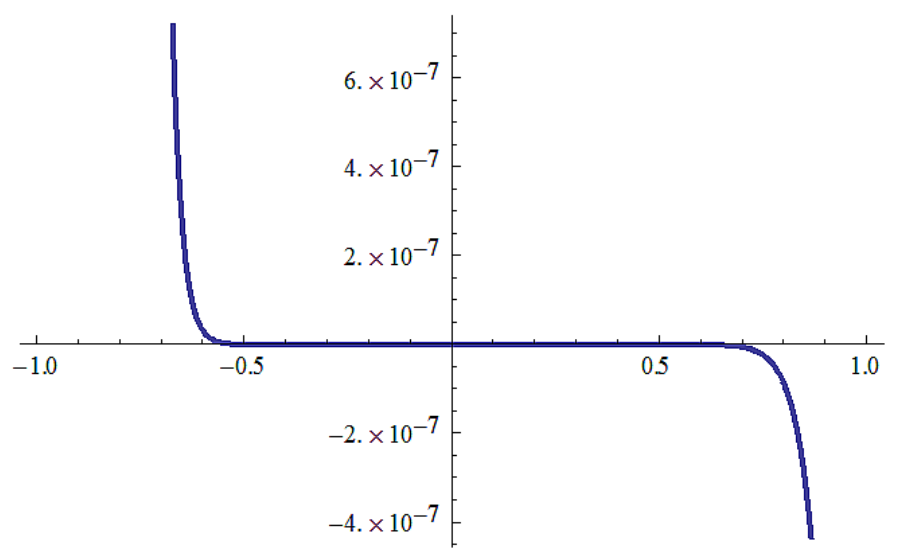

Fig. 1. Selection of function graphs

Source: own elaboration. 


\section{Matrix method}

Definition. Let $\mathbb{K}_{0}$ represent a subsemigroup of a semigroup $\mathbb{K}$ of the power series for which $b_{0}=0$ and $b_{1}=1$.

Semigroup $\mathbb{K}_{0}$ contains all the power series of the form

$$
x+a_{2} x^{2}+\cdots \text {. }
$$

Note. If function $f$ is odd and satisfies a quadratic equation, then also function $-f$ satisfies it too. If $f(f)=g$ then also $(-f(-f))=g$. This notice is a simple consequence of the definition of parity.

Theorem on uniqueness. The Kopociński equation in semigroup $\mathbb{K}_{0}$ has a uniquely determined solution.

Evidence. This theorem means that there is always one and only one solution. We shall give below an alternative method of calculating the coefficient of an analytic function with relation to the method referring to the partition described above. Both methods differ only in the way of deriving equations - the equations are the same. Consider the auxiliary matrix $A$ with an infinite number of elements

$$
\left(\begin{array}{cccc}
a_{11} & a_{12} & a_{13} & \ldots \\
a_{21} & a_{22} & a_{23} & \ldots \\
a_{31} & a_{32} & a_{33} & \ldots \\
\vdots & \vdots & \vdots & \ddots
\end{array}\right),
$$

where $a_{11}=1, a_{1 n}=b_{n}$ for $n \geq 2, a_{n i}=0$ for $i<n, a_{k n+1}=$ $\phi_{k n}\left(b_{1}, \ldots, b_{n}\right)$ for $1<k<n$. The function $\phi_{k n}$ is a unequivocally defined polynomial; its arguments are coefficients calculated before. Enumerating the coefficients of these polynomials is not easy. This $n$-th column of the matrix is used to calculate the coefficient $b_{n}$. In that column the coefficient $b_{n}$ occurs twice, and the remaining terms are well known and depend on the coefficients calculated previously. This column is thus a sequence:

$$
\left(b_{n}, \phi_{2 n}\left(b_{1}, \ldots, b_{n-1}\right), \ldots, \phi_{n-1 n}\left(b_{1}, \ldots, b_{n-1}\right), b_{n}, 0,0, \ldots\right) .
$$

Hence $a_{1 n}=a_{n n}=b_{n}$, this means that to calculate the coefficient $b_{n}$ we have linear equations in the form of $2 b_{n}=c_{n}$ where the size $c_{n}$ is determined by coefficients calculated in advance and free terms. This means that, firstly, a solution exists, and secondly, that there is only one which was to be proved. Of course, the Kopociński equation in semigroup $\mathbb{K}_{0}$ has a solution for any analytic function, and not only for the function $\ln (1+x)$. 
There is a third possible way of calculating the coefficients $b_{n}$ which also proves the theorem; it stems from the formula for the derivative of function composition. Just remember that the power series $k$-th derivative is equal to $k ! b_{k}$.

In the quadratic equation there naturally appears the product of Cauchy's sequences; if we multiply the power strings, their coefficient sequences are multiplied according to Cauchy's product. So if $a=\left\{a_{n}\right\}, b=\left\{b_{n}\right\}$ then the Cauchy product is equal

$$
\sum_{i=0}^{n} a_{n-i} b_{i}
$$

If so, $f(x)=\sum_{n=0}^{\infty} a_{n} x^{n}, g(x)=\sum_{n=0}^{\infty} b_{n} x^{n}$, then $f(x) g(x)=\sum_{n=0}^{\infty} c_{n} x^{n}$, where

$$
c_{n}=\sum_{i=0}^{n} a_{n-i} b_{i} .
$$

Let $b=\left(b_{0}, b_{1}, b_{2}, \ldots\right)$ and let $w_{n}$ mean the $n$-th row of the matrix $A$ shifted to the right by one place, that is $w_{n}=\left(0, a_{n 1}, a_{n 2}, \ldots\right)$. Of course $w_{n}=b_{n} b^{n}$, where $r e=b_{n} b^{n}$ is the $n$-th power of Cachy's series $b$. If, $b^{n}=\left(b_{n 0}, b_{n 1}, b_{n 2}, \ldots\right)$, then $g^{n}(x)=\sum_{k=n}^{\infty} b_{n k} x^{k}$. Hence:

$$
h(x)=\operatorname{gog}(x)=\sum_{k=1}^{\infty} b_{k} g^{k}(x) \text {; }
$$

so we have an equation $a_{k}=\sum_{i=1}^{k} b_{i} b_{i k}$ where $a_{n}$ is the $n$-th coefficient of expansion of a function $\ln (1+x)$ into series. So we have a recursive patterns in the form of: $b_{1 k}=b_{k}, b_{n+1 k}=\sum_{i=0}^{k} b_{n i} b_{k-i}$, where $k \in \mathbb{N}$ and $b_{n n+1}=$ $b_{n+1}$. Eventually $a_{n k}=b_{n} b_{n k}$. The equation for the $b_{k}$ coefficient has the form of $2 b_{k}=a_{k}-\sum_{i=1}^{k-1} a_{i k}$.

\section{Abstraction and identical objects}

Functions $f_{1}: X_{1} \rightarrow Y_{1}$ and $f_{2}: X_{2} \rightarrow Y_{2}$ are equivalent, if there are isomorphisms $\varphi_{1}: X_{1} \rightarrow X_{2}$ and $\varphi_{2}: Y_{1} \rightarrow Y_{2}$ such that, $\varphi_{2} \circ f_{1}=f_{2} \circ \varphi_{1}$, when $f_{1}=\varphi_{2}^{-1} \circ f_{2} \circ \varphi_{1}$. Equivalent functions work the same way in isomorphic collection. There is no difference whether we permute letters or if we permute numbers. If we talk about continuous functions, the isomorphisms are homeomorphisms of topological spaces. Functions $\ln (x)$ and $\ln (1+x)$ are equivalent. 
Let $\tau$ mean translation; $\tau(x)=x+1$; naturally, each translation is a square in the group of translations, for $\sigma(\sigma)=\tau$, where $\sigma(x)=x+\frac{1}{2}$. We solved the Kopociński equation in the form of $g \circ g=\ln \circ \tau$ which is equivalent to form $\circ \tau^{-1} \circ \tau \circ g \circ \tau^{-1}=\ln$. Introducing determination $f_{1}=g \circ \tau^{-1}$ we obtain $f_{1} \circ \tau \circ f_{1}=\ln$. If one changes the operation in a semigroup we obtain the exact equation in the form $f_{1} * f_{1}=\ln$, where $*$ is the new operation defined by the formula $f * g=f \circ \tau \circ g$. In a semigroup with the new operation with unity there is a translation $\tau^{-1}$ because $\tau^{-1}$ * $g=g$.

The law of one-and-a-half logarithm in the zero position, that is, after translation $\tau$ means that on the random variable function $g \circ \ln \circ \tau$ is applied. While the law of the one-and-a-half logarithm in neutral position, that is without moving, means that on the random variable function $\tau^{-1} \circ g \circ \ln \circ \tau$ applied.

Hypothesis. Let $\mathbb{K}_{a}$ mean a semigroup of power series with a positive radius of convergence; each series represents an analytic function, hence it is $\mathbb{K}_{a} \subset \mathbb{K}_{0}$. If $g \in \mathbb{K}_{0}$ is the solution for the Kopociński equation, then the question arises whether $g$ is an analytic function, so if $g \in \mathbb{K}_{a}$. This is a difficult problem in the field of analytic functions and power series. For if $g \notin \mathbb{K}_{a}$, then the equality $g$ o $g=f$ seems paradoxical. An analytic function is obtained from the series composition of zero-convergence. The nature of coefficients $b_{n}$ of function $g$ is unknown. They arise from the algebraic sum of very large numbers, so they can be themselves very large. This means that the radius of convergence of this series can be zero. This is the content of our assumptions. There is a full analogy to the equation $x^{2}=-1$ that has no solution in a multiplicative semigroups of real numbers, and has two solutions in a multiplicative semigroups of complex numbers. Mathematics knows many examples of contradictory equations that after the expansion of permissible objects have obtained generalized solutions.

We suppose that the natural logarithm without shift is not a square in the set of analytic functions with real coefficients. The argument in favor of this hypothesis is the following remark. If the areas of these features are the same, then there appears a contradiction. $\ln (1)=0$ and, $g(g(x))=\ln (x)$, so $g(1)=\alpha$, where $\alpha>0$, because it cannot be $\alpha \leq 0$. But $g(\alpha)=0$, so $g(g(\alpha))=g(0)$, hence the contradiction. 
A more general assertion. In semigroup $\mathbb{K}_{0}$ the generalized Kopociński equation has a unique solution. If the solution is not an analytic function, then there is a solution in the set $\mathbb{K}_{0}-$ a generalized solution.

The proof of this theorem - mutatis mutandis - is a repetition of the proof of the theorem about the existence of the equation $g \circ g=f$. If the function $g$ satisfies the equation $g^{m}=f^{n}$, then also for any $k \in \mathbb{N}$ the equality $g^{k m}=f^{k n}$ is true. This means that in the semigroup $\mathbb{K}_{0}$ fraction $1 / 3$ is equal to the fraction $7 / 21$. So it is enough to solve equations of form $g^{m}=f^{n}$ for numbers $n$ and $m$ that are relatively prime.

The aforementioned $\mathrm{G}$ semigroup of positive rational numbers with the operation of multiplication is in fact a commutative group - Abelian. This group is isomorphic to the group of $M$ sequences of integers with a finite number of terms different from zero. The isomorphism identifying the two groups is formed from the extension of a function associating to the first number ' $n$ ' a sequence that at the $n$-th location has number one, and zeros otherwise. $\mathrm{M}$ is essentially a module of such sequences above the ring of integers. So the prime number 2 has the number zero and the assigned string $(1,0,0, \ldots)$. Number 3 has the number 1 , so it is assigned to a string $(0,1,0,0, \ldots)$; prime number 5 has number 2 , so it corresponds to the string $(0,0,1,0,0, \ldots)$, and so on. Prime numbers are, in fact, a base for module $M$. The number 4 is identified with a string $2(1,0,0, \ldots)=(2,0,0, \ldots)$, number 6 is in this convention is a sum of a series of strings for 2 and 3 , that is $(1,1,0,0, \ldots)$. Rational number $15 / 8$ is a string: $-3(1,0,0, \ldots)+(0,1,0,0, \ldots)+(0,0,1,0,0, \ldots)=(-3,1,1,0,0, \ldots)$ This isomorphism identifies strings of integers of the specified property with the rational numbers. Multiplication of rational numbers is adding these strings; division on the other hand is subtraction. The index of integer number $n$ is what the sum of terms within its string is called. If so, $n=24=2^{3} \cdot 3$ then its index is the number four - the sum of the exponents of primes occurring in the factorization of this number. If the integer $n$ is the $k$-th power of another integer its index is divisible by $k$. The index 125 is divisible by 3 because $125=5^{3}$.

The concept of the index allows the hypothesis, which, if true, includes as a special case Fermat's Last Theorem. Fermat's Last Theorem says that if $\mathrm{n}$ is greater than or equal 3 , there are natural numbers different from 0 satisfying the equation $x^{n}+y^{n}=z^{n}$. In other words, if $F_{n} \subset \mathbb{N}$ denotes the set of natural numbers different from zero which are in the form $k^{n}$, where $n \in \mathbb{N}$, then if $\geq 3$ and $a, b \in F_{n}$ then $a+b \notin F_{n}$. 
The multiplicative semigroup $\mathbb{N}^{*}$ of natural numbers different from zero is isomorphic to the commutative semigroup of words in an infinite alphabet, in which there are prime numbers. In this semigroup, the uniqueness of factorization of natural numbers into prime factors is an obvious fact. The defined above index of an integer is equal to the length of the word that represents this number. Thus, each prime number is an index of 1 , the product of two primes has an index of 2, and so on. The number 0 does not have an index, and the number 1 has the index 0 .

The generalized hypothesis. If $\mathrm{n}$ is greater than equal to 3 and $x$ and $y$ are natural numbers different from zero, then the index of number $x^{n}+y^{n}$ is not divisible by $n$. If this generalized hypothesis is true, then it naturally follows Fermat's Theorem.

\section{Concluding remarks}

Mapping $\phi: \mathbb{K}_{0} \rightarrow \mathbb{K}_{0}$ described by the pattern $\phi(g)=g \circ f$, as mentioned above, covers the semigroup $\mathbb{K}_{0}$, that is, each element of the semigroup is a square. At the same time we have to stress that semigroup elements may have different radiuses of convergence and the radius of composition convergence of such series is dependent on factors - and it may vary.

The functions presented here can be looked at from a purely formal perspective. These are new non-elementary features that enhance the mathematical arsenal. On this occasion, we would like to recall a flat pseudo-mathematical joke. - I found a new smooth function. -Yes? And what did you do with it? - I differentiated it. Secondly, speaking about the probability theory we can discuss not only the logarithmic-normal disintegration, but also about disintegrations such as semi-logarithmic-normal, half-logarithmic-normal and so on. The one-and-a-half logarithm is the $g^{3}$ function. Furthermore, the article is a pictorial representation of a method of indefinite coefficients very useful in solving function equations.

\section{Bibliography}

Bödewadt U.T. (1944). Reeller Funktionen zur Iteration. Math. Z. 49, pp. 497-516.

Iga K. Continuous half-iterates of functions. Manuscript. URLs or http://math.stanford.edu/ iga http://math.pepperdine.edu/kiga/Papers/halfiter.ps.

Kneser H. (1950). Reelle analytische Lösungen der Gleichung $\psi(\psi(x))=e^{x}$ und verwandter Funktionalgleichungen. Journal für die reine und angewandte Mathematik 187, pp. 56-67. 
Kuczma M. (1969). Fractional iteration of differentiable functions. Annales Polonici Mathematici. Vol. 2. No. 22, pp. 217-227.

Paulsen W. (2016). Finding the natural solution to $f(f(x))=e^{x}$. The Korean Journal of Mathematics 24 (1), pp. 81-106.

Zhang W. (1997). PM functions. Their characteristic intervals and iterative roots. Annales Polonici Mathematici. Vol. 2. No. 65, pp. 119-128.

Zubrzycki S. (1962). Wzór rekurencyjny dla ilości partycji ograniczonych. Applicationes Mathematica 6 (2), pp. 231-234. 